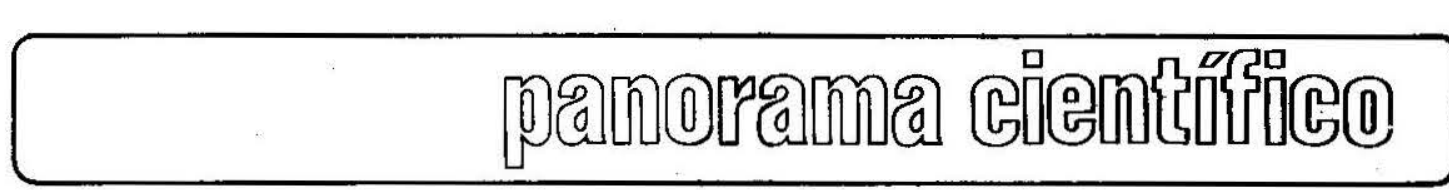

\title{
HONGOS VENENOSOS Y MICETISMOS
}

\author{
Wabdo Lazo \\ Departamento de Ciencias Ecológicas \\ Facultad de Ciencias, U. de Chile
}

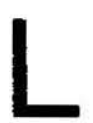

a ingestión de setas (callampas) veneno. sas produce-una enfermedad llamada "micetismo" que puede ser de diversos tipos, según sea el veneno que la ocasionó y las características del envenenamiento. Así se habla de Síndrome faloidínico (o faloídeo), parafaloidínico, giromítrico, alucinógeno, muscarínico, gastrointestinal, copr ínico, paxílico, etc. Los más graves son los síndromes faloidínico y parafaloidínico que frecuentemente son mortales.

En Europa y Estados Unidos, los hongos que ocasionan el Síndrome faloidínico són principalmente: Amanita phalloides, A. verna, A. virosa y algunas especies del género Galerina.

Este cuadro clínico llamado también "amanitismo" se caracteriza por presentar un período inicial asintomático que se prolonga por 8 a 12 horas o más después de la ingestión de las setas y que al cabo Ge ese tiempo hace un ostentoso debut caracterizado por un dolor abdominal agudo, vómitos y diarrea profusa; generalmente, un tratamiento con medidas de soporte produce el alivio del paciente, el cual puede sentirse bien durante algunas horas o dos a tres dias; las pruebas hepáticas, sin embargo, presentan una considerable alteración. Transcurrido este plazo el paciente empeora, puede presentar ictericia, colapso circulatorio, falla renal. La necrosis hepática suele ser masiva y el daño renal considerable.

Las toxinas culpables de este cuadro son las "falotoxinas" que son heptapéptidos cíclicos y que al parecer ocasionan la primera fase del envenenamiento, es decir, la de los síntomas intestinales agudos; las "amatoxinas" causan la fase hepato-renal, son octapéptidos y son 10 a 20 veces más potentes que las falotoxinas, la principal de ellas es la alfa amanitina. Las amatoxinas poseen una mayor afinidad por los hepatocitos y su efecto pernicioso es mayor porque la toxina secretada en la bilis y rẹciclada por via de la circulación entero-hepática. En el riñón, la toxina es reabsorbida del filtrado glomerular (1).

El diagnóstico precoz de la intoxicación es muy importante para instituir el tratamiento efectivo. El radioinmunoensayo para la amatoxi- na puede hacerse en muestras de jugo gástrico. sangre u orina, por supuesto el tratamiento debe ser iniciado antes de conocerse el resultado de este examen. La toxina se puede detectar en el aspirado duodenal hasta 36 horas después de la ingestión, posiblemente debido a la çirculación enterohepática y la aspiración del contenido duodenal puede evitar un mayor daño hepático. Las toxinas de la Amanita son fácilmente dializables y poseen una gran afinidad por el carbón. La hemodiálisis en carbón es un procedimiento efectivo para remover la toxina y los resultados clínicos obtenidos con ella son muy significativos (1).

Respecto al tratamiento quimioterápico, digamos que se recomienda la aplicación de dosis. elevadas de penicilina, fármaco que por tener mayor afinidad con las proteínas plasmáticas que las toxinas, las desplaza de ellas. Tambièn se recomienda la adíministración de silibina (Hepatrón), el componente más activo de la silimarina (Legalon) que es una mezcla de flavonas que se encuentran en el jugo del cardo sylibum ma rianum; también la administración de ácido tióctico y de dosis elevadas de vitamina $C$ (1).

Esquematizando el tratamiento, anotemos:

1. Lavado estomacal, dejar una sonda duodenal "in situ" y aspirar cada hora.

2. Rehidratación con solución de dextrosà al 5\%, solución salina y otros electrolitos.

3. Hemodiálisis por carbón, precozmente si el paciente está en mal estado o si comió gran cantidad de callampas.

4. Quimioterapia: ácido tióctico $200-500 \mathrm{mg}$ diarios por vía intravenosa durante las $\mathbf{2 4}$ horas: penicilina G $250 \mathrm{mg} / \mathrm{kg}$ diarios por vía intravenosa lenta; $20-40 \mathrm{mg}$ de dexametasona diarios por vía intravenosa; silibina, vitamina $C$.

Un tratamiento fácil de aplicar fue diseñado por Bastien (2), quien lo llamo "tratamiento de emergencia" y que es simple y barato. Consiste en la administración diaria por vía bucal de $1200 \mathrm{mg}$ de nifuroxacida y $1500 \mathrm{mg}$ de dihidroestreptomicina, además de $3 \mathrm{gr}$ de vitamina $\mathrm{C}$ diarios. Se debe administrar estos medicamentos durante tres días y alimentar al paciente sólo con sopa de 
zanahorias durante todo este tiempo.

Sobre el Síndrome muscarínico, señalemos que se caracteriza por su comienzo súbito (una a dos horas de incubación). Los pacientes pueden presentar confusión, excitación, jadeo, salivación, náuseas y vómitos, diarrea, pulso lento, pupilas contraídas (muscarina), pupilas dilatadas (atropina), temblores, debilidad, colapso y a veces muerte. Su tratamiento consiste en remover el contenido gastrointestinal por emesis y lavados seguidos de catarsis. Si los signos muscarínicos predominan, administrar $1-2 \mathrm{mg}$ de atropina por vía subcutánea y repetirla cada 30 minutos si se lo necesita. Si la toxicidad atropínica es la que predomina dar fisostigmina. Administrar sedativos barbitúricos para controlar la excitación. Administración de fluidos por vía oral y parenteral; tratar el shock.

En Chile, los micetismos eran, hasta hace pocos años, considerados como hechos que ocurrían esporádica y escasamente. Así, se había descrito intoxicaciones mortales por la ingestión de Lepiota locafiensis en 1935, 1943, 1946 (3).

Pero en 1967, se describió envenenamientos producidos por otra especie venenosa, la Amanita gemmata var. tóxica cuyas setas al ser ingeridas ocasionaron la muerte de dos personas. En los trece años siguientes, según la información que poseemos, no se comunicó el envenenamiento mortal de ninguna persona, aunque sí se describieron algunos envenenamientos no mortales. - Hasta que en 1980 y 1981, se comunicaron más de 30 casos de micetismos, de los cuales, por to menos, 7 pacientes fallecieron.

Hasta ahora no se han aislado todas las toxinas que contienen los carpórofos de $\boldsymbol{A}$. gemmata var. tóxica. Por la semejanza que existe entre el cuadro clínico que han presentado los pacientes en Chile y el "amanitismo" foráneo, presumimos que los venenos son los mismos o muy similares $y$, por lo tanto, posiblemente; el tratamiento que reseñamos antes para el amanitismo podría servir también para tratar a los intoxicados con $\boldsymbol{A}$. gemmata var. tóxica.

El cuadro clínico exhibido por los pacientes chilenos presenta dos modalidades: 1. Cuadro con predominio de sintomas neurológicos, es decir, midriasis, incoordinación motora, obnubilación, somnolencia, vértigos, alucinaciones visuales, ex. citación, ocasionalmente relajación de esfínteres, náuseas, vómitos, enrojecimiento de la piel. II. Cuadro con predominio de síntomas gastroentéricos, esto es, vómitos con o sin sangre, diarrea, intenso dolor abdominal, pruebas hepáticas marcadamente alteradas $y$ que revelan una profunda insuficiencia hepática (transaminasa oxalacítica $1800 \mathrm{u} / \mathrm{e}$, transaminasa pirúvica $1260 \mathrm{u} / \mathrm{e}$, bilirrubina directa $2,70 \mathrm{mg} \%$, bilirrubina total $5,39 \mathrm{mg}$ ) creatinina $4,23 \mathrm{mg} \%$, anemia, a veces respiración estertorosa, hipotonía muscular, gran midriasis. Las muertes registradas se produjeron en los pacientes con el cuadro g.gastrentérico. Ellos fueron casi siempre niños. Sus autopsias revelaron un prófundo daño hepático (3).

A. gemmata var tóxica presenta en la actualidad en nuestro país una amplia distribución que abarca desde Peñuelas (Valparaíso), alrededores de Santiago, Rancagua, Colchagua, Constitución, Concepción, Valdivia y Osorno, por lo que recomendamos la mayor prudencia a quienes gustan de salir a recolectar setas al campo para comerlas, ya que fácilmente pueden recoger una seta venenosa confundiéndola con una comestible y sufrir los desastrosos efectos que ocasiona su ingestión. Las toxinas de $\boldsymbol{A}$. gemmata var. tóxica son termoestables.

Hay además en Chile otros hongos cuyas fructificaciones pueden causar micetismos, aunque no tan peligrosos como los antes descritos. Estos hongos son: Paxillus involutus, Mycena pura, Panaeolus sphinctrinus, Panaeolus retirugis, Psilocybe semilanceata, Agaricus xanthodermus, Lepiota cristata, Hygrocybe cónica, Naematoloma sublateritium, Gyromytra antarctica, etc., y posiblemente muchos más, desconocidos aún para nosotros debido al limitado conocimiento actual de nuestra flora micológica.

Para terminar, repitamos 10 que anota G. Pacioni en su "Guía de Hongos" (4): "La dispersión incontrolada de grandes cantidades de sustancias tóxicas como los pesticidas (anticriptogámicos e insecticidas), utilizados en la agricultura, pueden convertir en venenosos al champiñón silvestre (Agaricus campestris), al Marasmius oreades, a Macrolepiota procera y a todos los hongos que entran en contacto con estos venenos. De la misma manera pueden hacerse tóxicos los hongos que crecen en zonas con - intenso tráfico automovilístico o de elevada concentración de contaminación, hongos que en condiciones naturales son óptimos comestibles. El hongo puede actuar como esponja $y$ concentrar en sus carpóforos metales peligrosísimos como el plomo y el mercurio, depositados en el aire por los tubos de escape 
de los automóviles o por la industria agrícola (fumigaciones) y que posteriormente han sido transportados al suelo por la lluvia".

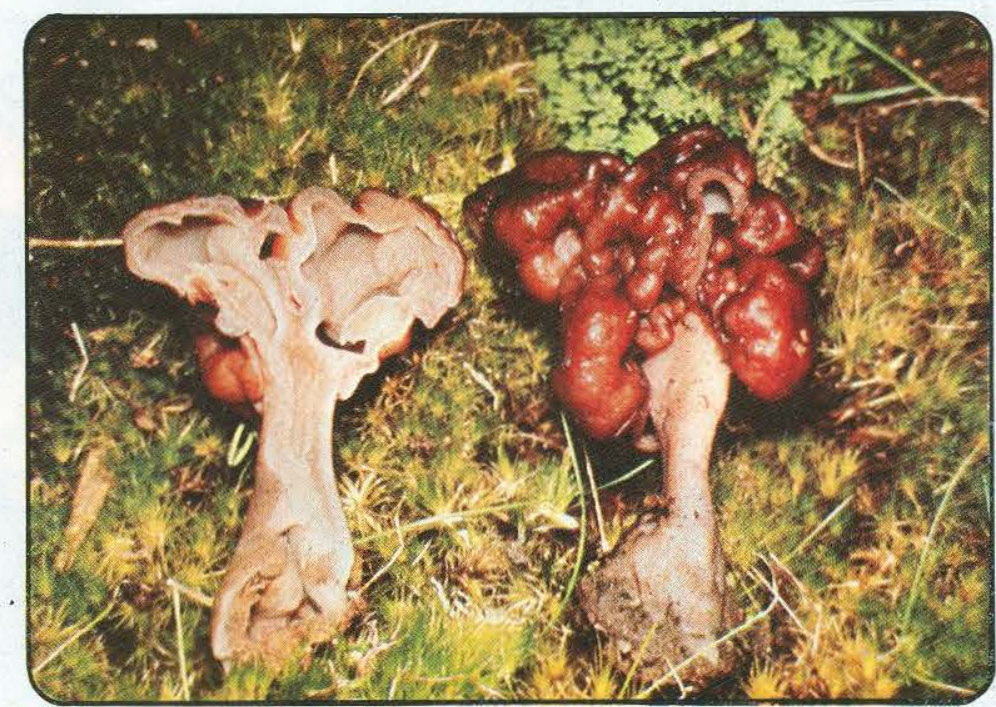

Gyromytra antarctica Rehim

Fructificación de $40-50 \mathrm{~mm}$ de altura. Sombrerillo de $20-35 \mathrm{~mm}$ de diámetro por $13-25 \mathrm{~mm}$ de alto, convoluto a cerebroide, castaño rojizo. Pedicelo de 25-50 $\mathrm{mm}$ de altura por $5-7 \mathrm{~mm}$ de diámetro, blanquizco grisáceo, glabro, hueco. Olor agradable. Sabor agradable.

Esporas de $20-24 \times 11-13$ u. hialinas, lisas, elip- soideo alargadas. Ascos de $280-320 \times 16-20 \mathrm{u}$, octosporados, cilíndricos, no amiloides. Paráfisis simples, pluriseptadas, contienen pigmento castaño rojizo.

Habitat: se desarrolla en el suelo, entre los restos vegetales en descomposición, en madera somipodrida.

Toxicidad: síndrome giromítrico (síntomas gastrointestinales y del sistema nervioso).

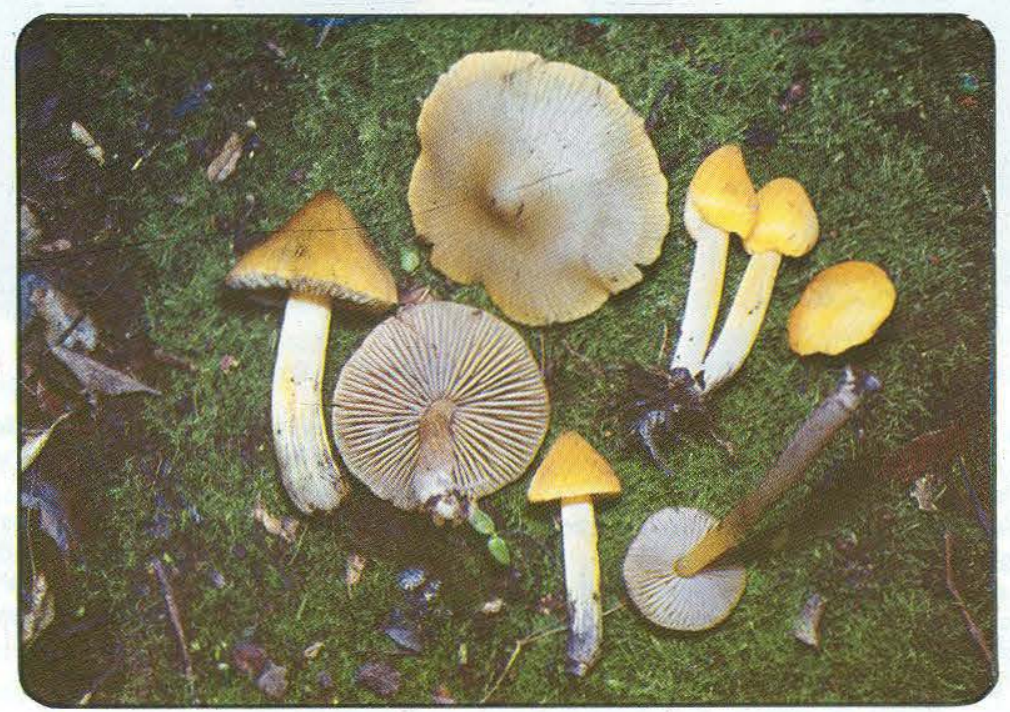

Hygrocybe cónica (Scop. ex Fr. Kummer)

Sombrerillo de 43-70 mm de diámetro, amarillo ámbar, cónico, después convexo, brillante sedoso cuando está seco, estriado en los ejemplares viejos. Lamelas blanco amarillentas que después ennegrecen, anchas, sub-libres. Pedicelo de 60-98 × 7-12 mm, blanquizco con el ápice amarillo, seco. La contextura exuda un látex acuoso cuando está fresca. Toda la superficie y la carne al romperse toman un color gris negro, que también lo adquieren al secarse. Sabor discreto. Inodoro.

Esporas de 10.5-13.2 × 5.5-7.5 u, elipsoides u ovoides, hialinas. Basidios de 38,5-52 x 9.5-10.5 u, hialinos, tetrasporados generalmente. Hifas con fíbulas.

Habitat: en el suelo, entre las hojas secas, dentro del bosque.

Toxicidad: produce un envenenamiento de tipo gastrointestinal. 


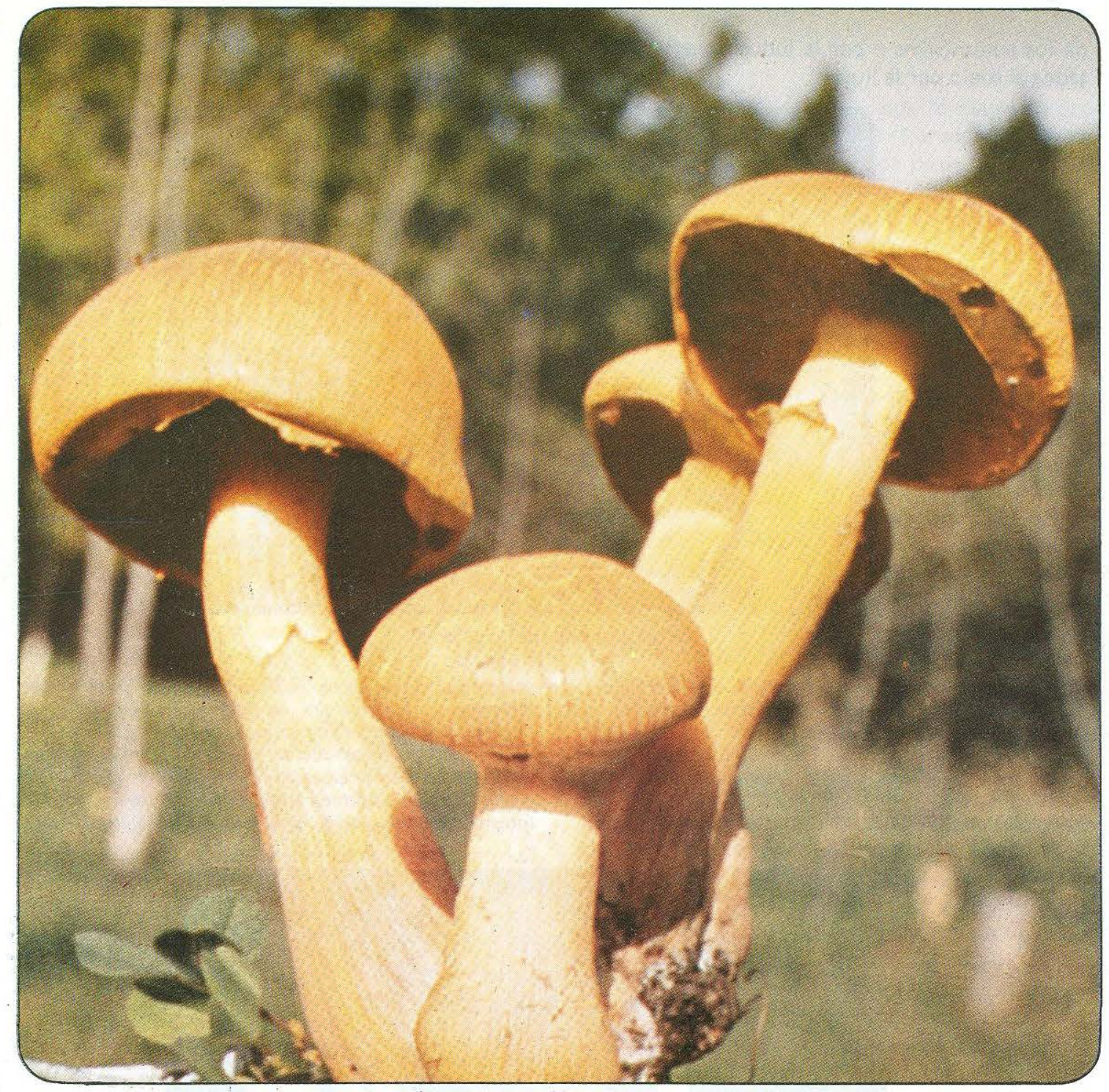

\section{Gymnopilus spectabilis (Fr.) Smith}

Sombrerillo de 40-250 mm de diámetro, naranja dorado a castaño naranja, convexo, seco, con la superficie fragmentada en pequeñas escamas fibrosas en la madurez. Pedicelo de 50-200X10-30 mm concoloro a sombrerilio, algo fibriloso, recio, grueso, duro, compacto, con un anillo membranoso delgado. Contextura gruesa, compacta, carne amarilla. Sabor ligeramente amargo. Olor ligero. Esporada pardo ferruginosa.

Esporas de 8-10 x 4.5-5u, color roya oscuro. ovoides a elipsoides. Basidios de 24-29 × 6.5-8u, hialinos a pálido amarillentos, bi y tetrasporados. Hifas con fibulas.

Habitat: se desarrolla en los aromos, eucaliptus, algunas veces en pinos. Muy abundante en Chile central.

Toxicidad: una variedad de esta especie puede producir intoxicaciones del sistema nervioso del tipo alucinógeno.

\section{BIBLIOGRAFIA}

1 Editorial. Mushroom poisoning. Lancet 1980. ii.: $351-352$.

2 Dumont, A.; Chennebault, J.; Alquier, P.; Jardel, H. Management of Amanits phalloides poisoning by Bastien's regimen. Lancet 1981. i: 722.
3 Lazo. W. Hongos venenosos en Chile. Bol. Inst. Salud Pub. Chile, 1982. 23. (1 y 2): 122-126.

4 Paccionni, G. Guía de Hongos. Ed. Grijalbo 1982. Barcelona, $523 \mathrm{pp}$. 